\title{
Evaluation of the Effectiveness of Laser System Control the Direction of Scattering Damaging Elements of the Projectile with the Defeat of Air Targets
}

\author{
Vladimir V. Butuzov, Yuriy L. Koziratsky, \\ Dmitri V. Prohorov and Roman G. Hilchenko* \\ Military Education and Research Centre of Military-Air Forces \\ «Military-Air Academy \\ Named After Professor N.E. Zhukovsky and Yu.A. Gagarin» \\ 54a Starykh Bolshevikov Str., Voronezh, 394064, Russia
}

Received 23.11.2017, received in revised form 10.10.2018, accepted 04.01.2019

Justified method of destruction of the aircraft-interceptor gun from the helicopter using the shell with the laser system control the direction of scattering damaging elements, an assessment of the effec-tiveness of this system.

Keywords: conflict "the helicopter - aircraft interceptor", laser control system, submunitions scattering, assessment of the effectiveness.

Citation: Butuzov V.V., Koziratsky Yu.L., Prohorov D.V., Hilchenko R.G. Evaluation of the effectiveness of laser system control the direction of scattering damaging elements of the projectile with the defeat of air targets, J. Sib. Fed. Univ. Eng. technol., 2019, 12(3), 266-274. DOI: 10.17516/1999-494X-0135.

(c) Siberian Federal University. All rights reserved

This work is licensed under a Creative Commons Attribution-NonCommercial 4.0 International License (CC BY-NC 4.0).

* Corresponding author E-mail address: vvbutuzov@yandex.ru 


\title{
Оценка эффективности лазерной системы управления \\ направлением разлета поражающих элементов снаряда \\ при поражении воздушных целей
}

\author{
В.В. Бутузов, Ю.Л. Козирацкий, \\ Д.В. Прохоров, Р.Г. Хильченко \\ Военный учебно-научный цеентр Военно-воздушных сил \\ «Военно-воздушная академия \\ имени профессора Н.Е. Жуковского и Ю.А. Гагарина» \\ Россия, 394064, Воронеж, ул. Старых Большевиков, 54 а
}

Обоснован способ поражения авиационных комплексов перехвата пушечным вооружением вертолета с использованием снаряда с лазерной системой управления направлением разлета поражающих элементов, произведена оценка эффективности этой системы.

Ключевые слова: конфликт «вертолет - авиационный комплекс перехвата», лазерная система управления, разлет поражающих элементов, оченка эффективности.

\section{Введение}

Анализ войн и военных конфликтов последних лет показывает, что вопросы защиты вертолетов при решении ими различных боевых задач достаточно актуальны. При этом особую опасность для них представляют не только системы маловысотной ПВО, но и авиационные комплексы перехвата (АКП) [1]. Для борьбы с такими комплексами на вооружении современных вертолетов имеются средства ракетного и пушечного вооружения. Боеприпасы, используемые в пушечных установках вертолета, в сравнении с ракетным вооружением экономически выгоднее в использовании, однако их точность в силу отсутствия систем наведения существенно ниже точности управляемых ракет. Поэтому задача повышения эффективности применения пушечного вооружения вертолета по АКП представляется актуальной.

Целью настоящей статьи является обоснование способа поражения АКП пушечным вооружением вертолета с использованием снаряда (с осколочно-фугасной боевой частью (БЧ) с направленным полем разлета осколков) с лазерной системой управления направлением разлета поражающих элементов (ПЭ) и оценка эффективности этой системы.

Прототипом предлагаемого способа служит способ, предложенный в $[2,3]$. Принципиальным отличием данного способа является полная автономность снаряда по принципу «выстрелил - забыл». Способ обеспечивает компенсацию ошибок наведения пушечной установки на цель (АКП) за счет выбора на траектории полета снаряда точки его подрыва. Для этой цели в состав снаряда включается дистанционный или неконтактный взрыватель [4], оценивающий пространственное положение снаряда относительно цели в процессе полета. В качестве снаряда можно рассматривать боеприпасы с осколочно-компрессионной боевой частью с направленным полем разлета осколков, например, за счет поворота снаряда перед подрывом БЧ вокруг продольной оси в сторону цели.

Основа неконтактного взрывателя - лазерная система управления направлением разлета ПЭ. Основные элементы системы: полупроводниковый лазер (ППЛ), матричный фотоприем-

$$
-267-
$$


ник (МФП), блок оценки дальности до цели, блок оценки угловых координат, блок определения угла сближения, при котором должна формироваться команда на отстрел. В процессе полета снаряда и его вращения вокруг своей оси со скоростью $n(t)$ полупроводниковый лазер формирует в направлении цели непрерывное лазерное излучение, которое, отражаясь от АКП, попадает на вход матричного фотоприемника в виде импульса оптического излучения. При этом конструктивное размещение ППЛ в снаряде и формируемое лазерное излучение с заданной расходимостью луча с учетом вращения снаряда вокруг своей оси должны обеспечивать угловой рабочий сектор $2 \alpha_{y c m}$ (где $\alpha_{y c m}$ - конструктивно установленный на снаряде угол отстрела поражающих элементов). По принимаемым матричным фотоприемникам в моменты времени $t_{1}$ и $t_{2}$ импульсам (рис. 1) производится оценка текущей дальности до АКП (от снаряда) $D_{1}$ и $D_{2}$ [5], измерение текущих угловых координат на АКП (относительно снаряда) $\alpha_{1}, \alpha_{2}$ [6-8] и определение угла сближения, при котором должна формироваться команда на отстрел.

\section{Определение момента подрыва боевой части снаряда}

Ограничения и допущения, принимаемые при разработке и оценке эффективности данного способа:

1. Стрельба (поражение) осуществляется на встречных курсах.

2. Значения скоростей снаряда и АКП, а также период оборота (вращения) снаряда постоянны.

3. Минимальное количество измерений -2 .

4. При попадании АКП в зону активной локации системы должно выполняться условие: $n(t) \cdot T \geq 2$, где $T$ - период оборота (вращения) снаряда.

С учетом вышесказанного скорости изменения угла сближения и дальности (расстояние от ППЛ снаряда до АКП) можно представить в виде отношений:

$$
\dot{\alpha}=\frac{\alpha_{2}-\alpha_{1}}{T} ; \dot{D}=\frac{D_{2}-D_{1}}{T},
$$

где $\alpha_{1}, \alpha_{2}$ - угловые координаты АКП (относительно снаряда), измеренные в моменты времени $t_{1}$ и $t_{2}$ соответственно; $D_{1}, D_{2}$ - дальности до АКП, измеренные в моменты времени $t_{1}$ и $t_{2}$ соответственно.

Введем в рассмотрение время инерционности формирования взрыва $\tau_{n}$ и скорость полета облака поражающих элементов $v_{n э}$.

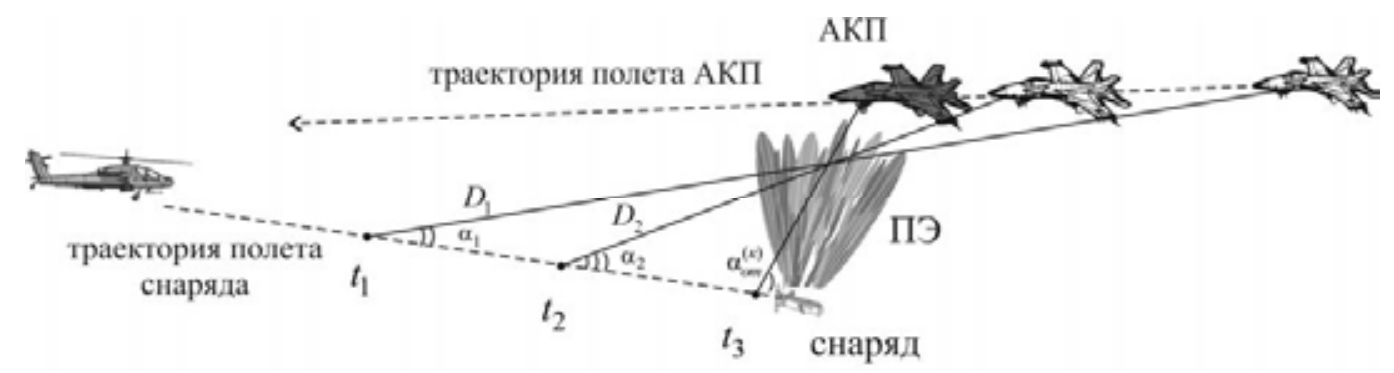

Рис. 1

Fig. 1 
Тогда угол сближения, при котором должна формироваться команда на отстрел,

$$
\alpha_{o m}^{(\kappa)}=\alpha_{y c m}-\frac{\alpha_{2}-\alpha_{1}}{T}\left(\frac{D_{n э}}{v_{n \ni}}+\tau_{n}\right),
$$

где $\alpha_{y c m}-$ конструктивно установленный на снаряде угол отстрела поражающих элементов; $D_{n э}$ - дальность полета поражающих элементов от снаряда до АКП.

Дальность полета поражающих элементов от снаряда до АКП $D_{n э}$ можно определить как

$$
D_{n э}=D_{2}+\dot{D}\left(t_{3}-t_{2}\right),
$$

где $t_{3}$ - момент времени формирования облака поражающих элементов.

Если последнее измерение дальности было выполнено в момент времени $t_{2}$, а $t_{3}-t_{2}=T$, то

$$
D_{n \ni}=D_{2}+\dot{D}\left(t_{3}-t_{2}\right)=D_{2}+\dot{D} T=2 D_{2}-D_{1}
$$

С учетом выражений (1), (3) и зависимости $\alpha$ и $D$ от времени получим следующее соотношение:

$$
\alpha_{o m}^{(\kappa)}(t)=\alpha_{y c m}-\dot{\alpha}(t)\left(\frac{2 D(t)-D(t-T)}{v_{n э}}+\tau_{n}\right),
$$

позволяющее, задаваясь параметрами снаряда, рассчитывать по результатам оценки текущих дальности и угла сближения по двум текущим измерениям в моменты времени $t-T$ и $t$ угол сближения, при котором должна формироваться команда на отстрел.

При рассмотрении эпизода стрельбы пушечным вооружением вертолета по АКП на встречных курсах проведены расчеты для следующих исходных данных: скорость снаряда $v_{c}=1000 \mathrm{~m} /$; скорость АКП $v_{a}=250 \mathrm{~m} /$; дальность выстрела 500 м; ошибка прицеливания 0,5 град; $v_{n э}=2000 \mathrm{м} / \mathrm{c} ; \tau_{n}=0,01 \mathrm{c} ; \alpha_{y c m}=60$ град; $T=4$ мс.

На рис. 2 представлены графики зависимостей угла сближения, при котором должна формироваться команда на отстрел, и текущего угла сближения снаряда с целью от времени.

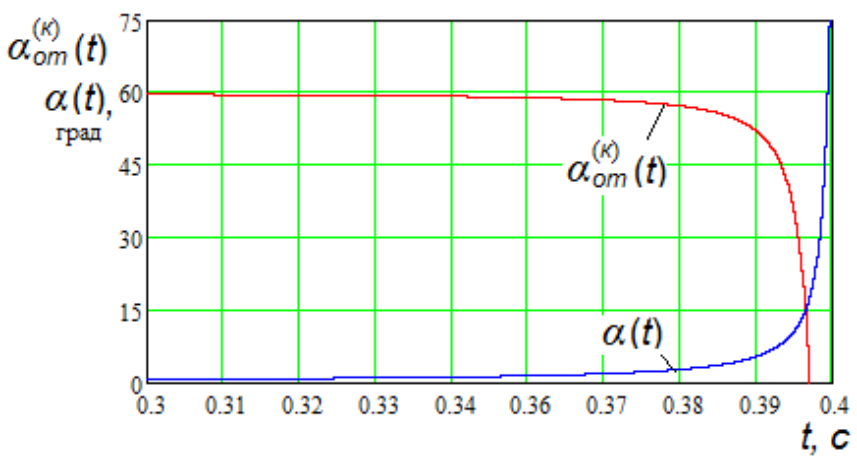

Рис. 2

Fig. 2 
В соответствии с полученными результатами угол сближения, при котором должна формироваться команда на отстрел, при вышеуказанных исходных данных должен быть равен 15 град, а время отстрела - 0,397 с (пересечение зависимостей на рис. 2).

\section{Задача прогнозирования угла сближения снаряда и АКП}

Однако очевидно, что расчеты с использованием выражения (4) будут давать существенные ошибки, обусловленные значимым увеличением скорости изменения угла сближения $\dot{\alpha}(t)$ по мере приближения снаряда к цели. Поэтому для повышения точности определения угла сближения $\alpha_{o m}^{(k)}(t)$ необходимо уменьшение периода формирования зондирующих импульсов ППЛ $T$. При этом существуют определенные ограничения в возможности уменьшения периода формирования зондирующих импульсов ППЛ $T$, обусловленные периодом (оборота) вращения снаряда и его конструкционными особенностями. Поэтому возникает задача прогнозирования угла сближения $\alpha(t)$.

Для решения этой задачи примем допущение о том, что ошибка прицеливания на протяжении полета снаряда остается постоянной (т.е. траектории полетов снаряда и АКП параллельны). Тогда, решая несложную геометрическую задачу, получим следующее соотношение:

$$
\frac{d \alpha(t)}{d t}=-\frac{1}{D(t)} \frac{d D(t)}{d t} \cdot \operatorname{tg} \alpha(t)
$$

Решая данное дифференциальное уравнение для начальных условий $\alpha\left(t_{0}\right), D\left(t_{0}\right)$, где $t_{0}-$ момент выстрела, получим следующее выражение для прогноза угла сближения снаряда и АКП:

$$
\alpha_{n p}(t)=\arcsin \left\{\frac{\sin \alpha\left(t_{0}\right) \cdot D\left(t_{0}\right)}{D(t)}\right\} .
$$

Ввиду того, что траектория снаряда нелинейная, возникают расхождения полученной прогнозной зависимости с реальной $\alpha(t)$ (особенно на конечном участке, рис. 3). Кроме того, необходим также прогноз дальности $D(t)$.

Для аппроксимации угла сближения по зондирующим импульсам ППЛ с фиксированным периодом $T\left(t>t_{k}\right)$ может быть получено следующее выражение:

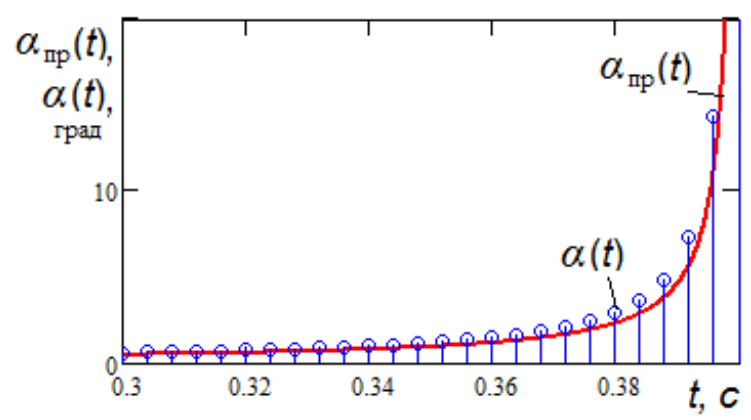

Рис. 3

Fig. 3 


$$
\alpha_{n p}(t)=\arcsin \left\{\frac{\sin \alpha\left(t_{k}\right) D\left(t_{k}\right)}{D\left(t_{k}\right)+\frac{D\left(t_{k}\right)-D\left(t_{k}-T\right)}{T}\left(t-t_{k}\right)}\right\}
$$

где $t_{k}=0, T, 2 T .$. - время приема зондирующих импульсов; $t$ - текущее время.

Результаты расчетов с использованием выражения (7) представлены на рис. 4.

Прогнозирование угла сближения $\alpha(t)$ с учетом его аппроксимации в текущий момент времени (выражение (7)) позволяет рассчитывать время отстрела снаряда следующим образом:

$$
t_{n p}=t_{n}-\frac{D_{n \ni}}{v_{\ni}}-\tau_{n}
$$

где $t_{n}$ - прогнозируемое время поражения цели при $\alpha(t)=\alpha_{y c m}, \tau_{n}=0$ и $\tau_{n э}=D_{n э} / v_{n э}=0$ (рис. 5).

Из выражения (7) получим

$$
t_{n}=t_{k}+T \frac{D\left(t_{k}\right)\left[\sin \alpha\left(t_{k}\right)-\sin \alpha_{y c m}\right]}{\left[D\left(t_{k}\right)-D\left(t_{k}-T\right)\right] \sin \alpha_{y c m}} .
$$

В выражении (8) дальность полета поражающих элементов от снаряда до АКП в момент подрыва снаряда может быть определена следующим образом:

$$
D_{n \ni}\left(t_{n p}\right)=D\left(t_{k}\right)+\frac{D\left(t_{k}\right)-D\left(t_{k}-T\right)}{T}\left(t_{n p}-t_{k}\right) .
$$

Решая систему уравнений (8), (9), (10), окончательно получим выражение для определения времени подрыва снаряда на основе прогноза дальности до цели и угла сближения снаряда и цели:

$$
t_{n p}\left(t_{k}\right)=\frac{t_{k}+T \frac{D\left(t_{k}\right)\left[\sin \alpha\left(t_{k}\right)-\sin \alpha_{y c m}\right]}{\left[D\left(t_{k}\right)-D\left(t_{k}-T\right)\right] \sin \alpha_{y c m}}-\frac{D\left(t_{k}\right)}{v_{n \ni}}+t_{k} \frac{D\left(t_{k}\right)-D\left(t_{k}-T\right)}{T v_{n \ni}}-\tau_{n}}{1+\frac{D\left(t_{k}\right)-D\left(t_{k}-T\right)}{T v_{n \ni}}}
$$

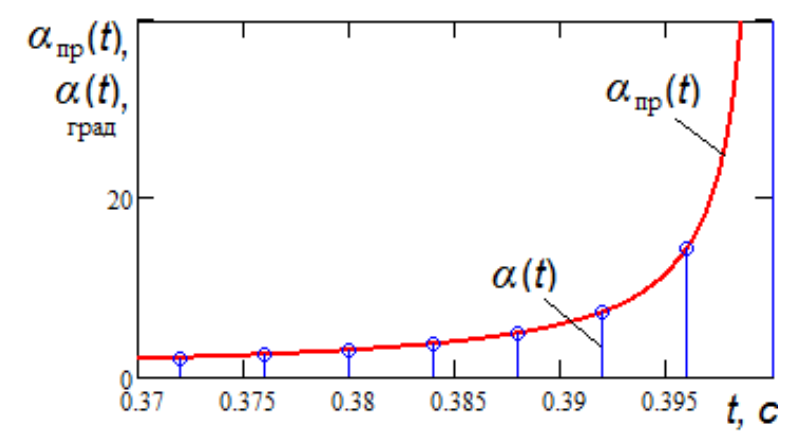

Рис. 4

Fig. 4 


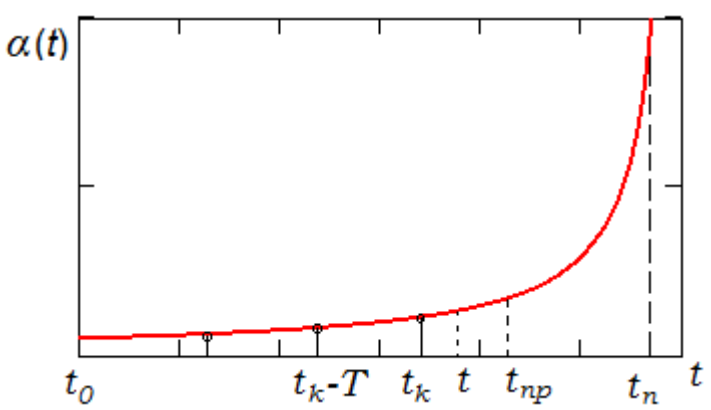

Рис. 5

Fig. 5

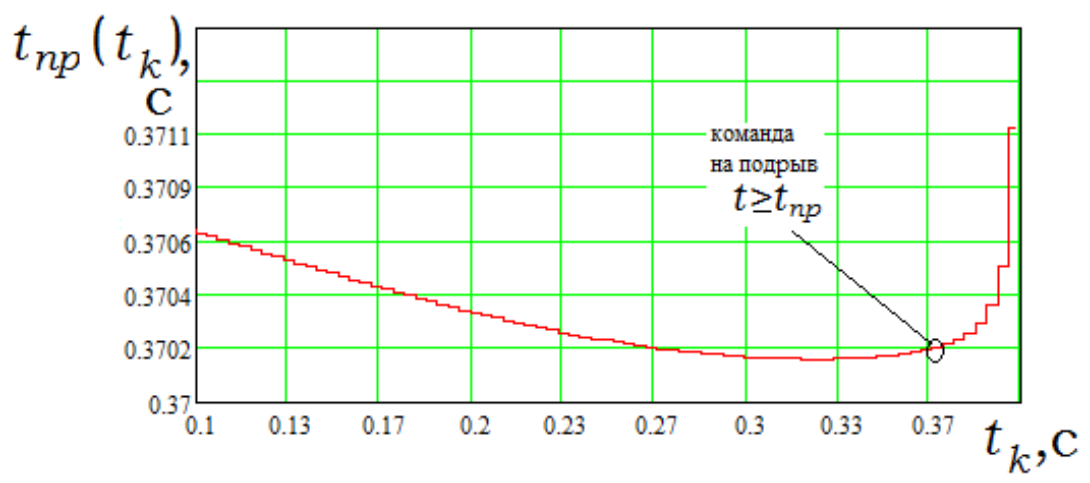

Рис. 6

Fig. 6

В соответствии с выражением (11) для указанных выше исходных данных были произведены расчеты и получен график зависимости прогнозируемого времени подрыва снаряда от времени приема зондирующих импульсов (рис. 6).

На рис. 7 представлена функциональная схема имитационной модели, реализующей вычисления в соответствии с полученным выражением (11).

\section{Имитационная модель процесса}

\section{определения времени подрыва боевой части \\ снаряда и угла сближения снаряда и цели}

Предположим, что ошибки определения угла сближения и текущей дальности в моменты времени $t_{k}=0, T, 2 T \ldots$ распределены по нормальному закону со среднеквадратическим отклонением (СКО) 0,02 рад и 0,2 м соответственно.

В результате имитационного моделирования (число измерений 500) были получены статистические значения ошибок оценки момента времени, при котором должна формироваться команда на отстрел, представленные в виде гистограммы на рис. 8. При нормально распределенных ошибках оценок угловых координат и дальности математическое ожидание ошибки оценки времени отстрела $m_{\Delta t_{n p}}$ составило $-3,7 \cdot 10^{-3} \mathrm{c}$, а СКО $\sigma_{\Delta t_{n p}}-4,5 \cdot 10^{-3} \mathrm{c}$. 


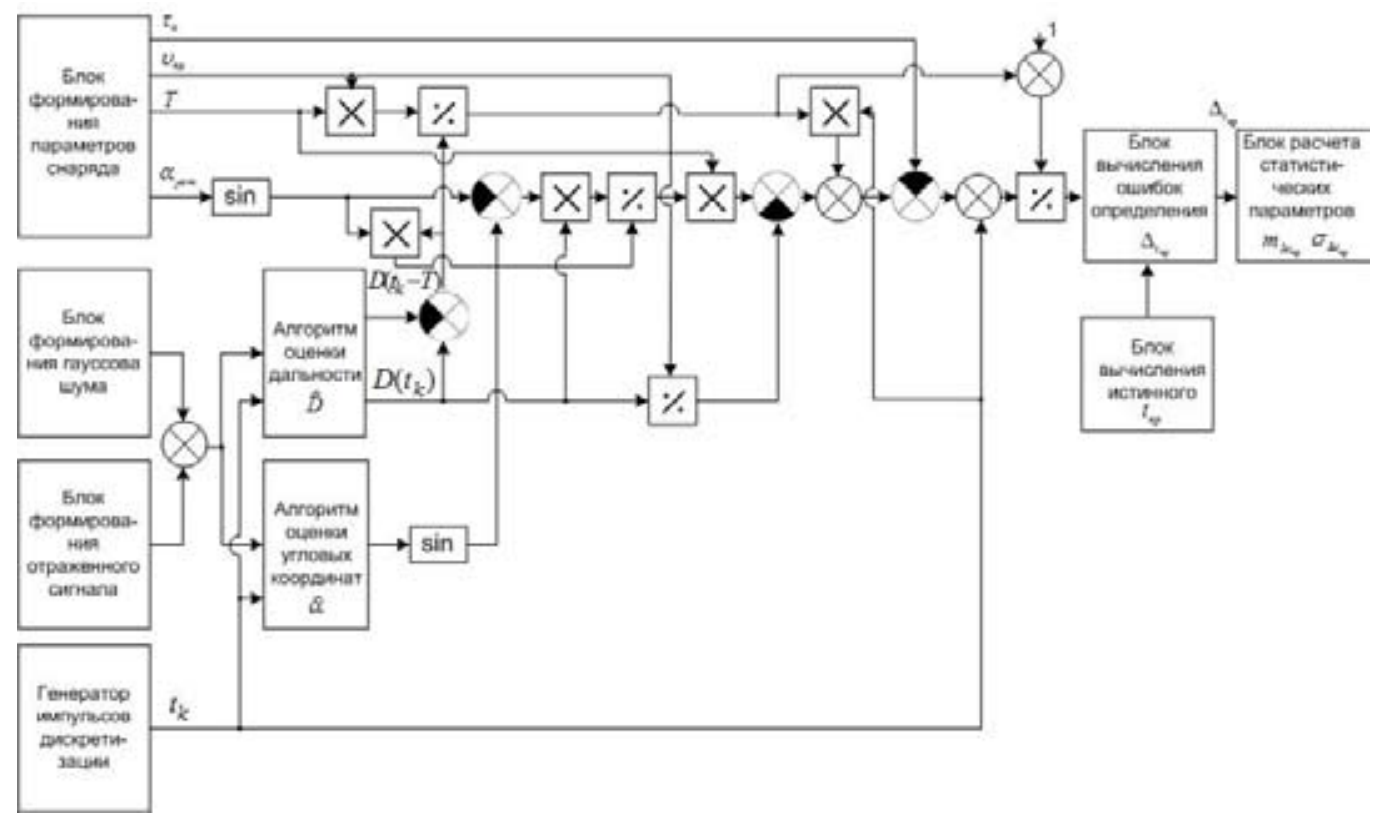

Рис. 7

Fig. 7

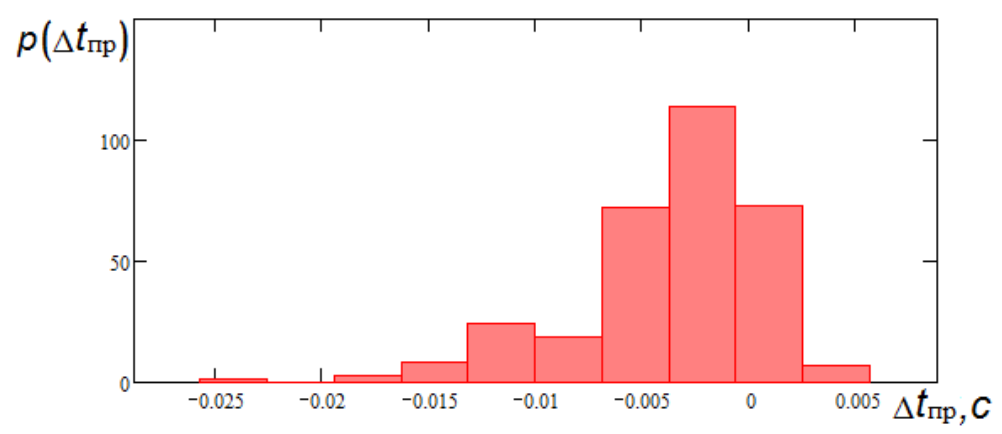

Рис. 8

Fig. 8

\section{Заключение}

Таким образом, обоснован способ поражения авиационных комплексов перехвата пушечным вооружением вертолета с использованием снаряда с лазерной системой управления направлением разлета поражающих элементов. Получено выражение для определения времени подрыва снаряда на основе прогноза дальности до цели и угла сближения снаряда и цели. Разработана имитационная модель оценки момента времени, при котором должна формироваться команда на отстрел поражающих элементов в направлении цели. Произведена оценка эффективности предложенной лазерной системы управления. 


\section{Список литературы}

[1] Козирацкий Ю.Л., Бутузов В.В., Хильченко Р.Г. Живучесть вертолетных комплексов армейской авиации как один из основных факторов повышения боевых возможностей ударных вертолетных групп, Военная мысль, 2017, 4, 19-25 [Kozirazkiy Yu.L., Butuzov V.V., Hilchenko R.G. Survivability of helicopter complexes for army aviation as one of the main factors of increasing the combat capabilities of strike helicopter groups, Voennaya mysl, 2017, 4, 19-25 (in Russian)].

[2] Козирацкий Ю.Л., Кулешов П.Е., Прохоров Д.В., Хильченко Р.Г. Лазерная система управления направлением разлета поражающих элементов снаряда при поражении дистанционнопилотируемого летательного аппарата, Вестник ВBA, 2015, 3(24), 166-169 [Kozirazkiy Yu.L., Kuleshov P.E., Prohorov D.V., Hilchenko R.G. The laser system control the direction of submunitions scattering of the projectile with the defeat of remotely piloted aircraft, VestnikVVA, 2015, 3(24), 166-169 (in Russian)].

[3] Бутузов В.В., Козирацкий Ю.Л., Кулешов П.Е., Прохоров Д.В. и др. Патент на изобретение RU № 2610734 C2, F42B 15/01, Способ поражения малогабаритных летательных аппаратов. Роспатент, 2015 [Butuzov V.V., Kozirazkiy Yu.L., Kuleshov P.E., Prohorov D.V., Method of destruction of small aircraft, Patent for invention RU No. 2610734 C2, F42B 15/01, 2015, (in Russian)].

[4] Бабкин А.В., Сухарь И.М., Велданов В.А., Грязнов Е.Ф. и др. Средства поражения и боеприпасы, М.: Издательство МГТУ им. Н.Э. Баумана, 2008, 982 с. [Babkin A.V., Suhar I.M. Veldanov V.A., Gryaznov E.F. Weapons and ammunition, Moscow: Publishing house of MGTU named after Bauman, 2008, 982 p. (in Russian)].

[5] Козирацкий Ю.Л., Прохоров Д.В., Курьянов И.Ю., Кулешов П.Е. Алгоритм оценки дальности до движущегося воздушного объекта, Радиотехника, 2014, 9, $73-75$ [Kozirazkiy Yu.L., Prohorov D.V., Kuryanov I.Yu., Kuleshov P.E. The algorithm for estimating the distance to a moving air object, Radiotechnics, 2014, 9, 73-75 (in Russian)].

[6] Козирацкий Ю.Л., Прохоров Д.В., Казаков В.С., Кусакин А.В. Алгоритм совместной оценки положения и времени прихода пуассоновского изображения цели и его эффективность в условиях помех, Радиотехника, 2012, 5, 102-106 [Kozirazkiy Yu.L., Prohorov D.V., Kazakov V.S., Kusakin A.V. The algorithm combines estimates of the position and time of arrival of the Poisson image goals and its effectiveness in conditions of interference, Radiotechnics, 2012, 5, 102-106 (in Russian)].

[7] Козирацкий Ю.Л., Паринов М.Л., Прохоров Д.В., Кильдюшевский В.М. Модель процесса определения координат наземных объектов, Радиотехника, 2012, 5, 61-64 [Kozirazkiy Yu.L., Parinov M.L., Prohorov D.V., Kildyushevskiy V.M. Model of the process of definition of coordinates of ground objects, Radiotechnics, 2012, 5, 61-64 (in Russian)].

[8] Козирацкий А.Ю., Кулешов П.Е., Паринов М.Л. Модель процесса функционирования кодового фотоприемника в режиме определения угловых координат источника излучения, Информатика: Проблемы, методология, технологии: Материалы VI международной научно-методической конференции. Воронеж: ВГУ, 2006, 178-181 [Kozirazkiy Yu.L., Kuleshov P.E., Parinov M.L. The model of the functioning of the code of the sensor in the mode of determining the angular coordinates of the radiation source, Informatics: Problems, methodology, technologies, Proceedings of the VI international scientific-methodical conference, 2006, 178-181 (in Russian)]. 